\title{
Erratum to: Fast Computing of Conformal Mapping and Its Inverse of Bounded Multiply Connected Regions onto Second, Third and Fourth Categories of Koebe's Canonical Slit Regions
}

\author{
Ali W. K. Sangawi ${ }^{1,2}$. Ali H. M. Murid ${ }^{3,4}$. \\ Lee Khiy Wei ${ }^{3,4}$
}

Published online: 2 April 2016

(C) Springer Science+Business Media New York 2016

\section{Erratum to: J Sci Comput DOI 10.1007/s10915-016-0171-3}

The authors regret that few corrections were inadvertently omitted from the original paper. It is now included with this erratum.

We would like to note the following corrections:

- Under Section 2, Line 36, 3rd integral operator $\mathbf{N}^{*}$ should be like

$$
\mathbf{N}^{*} \psi(t)=\int_{J} N^{*}(t, s) \psi(s) d s, \quad t \in J .
$$

- Under Section 7, "Numerical Implementation" Lines 10-15 should change to The integral equation (49) can be written as

$$
\int_{J}-N_{k}(s, t) d t S^{\prime}(t)+\int_{J} N^{*}(s, t) S^{\prime}(s) d s+\mathbf{J} S^{\prime}=\tilde{\psi}(t),
$$

The online version of the original article can be found under doi:10.1007/s10915-016-0171-3.

$\bowtie \quad$ Ali W. K. Sangawi

ali.kareem@univsul.edu.iq

Ali H. M. Murid

alihassan@utm.my

Lee Khiy Wei

leekhiywei@gmail.com

1 Department of Mathematics, Faculty of Science and Science Education, School of Science, University of Sulaimani, 46001 Sulaimani, Kurdistan, Iraq

2 Department of Computer, College of Basic Education, Charmo University, 46023

Chamchamal Sulaimani, Kurdistan, Iraq

3 Department of Mathematics, Faculty of Science, Universiti Teknologi Malaysia, 81310 Johor Bahru, Johor, Malaysia

4 UTM Centre for Industrial and Applied Mathematics, Ibnu Sina Institute for Scientific and Industrial Research, 81310 Johor Bahru, Johor, Malaysia 
where $N_{k}(s, t)=\frac{1}{\pi} \operatorname{Im}\left(\frac{z^{\prime}(t)}{z(t)-z(s)}\right)$,

Discretizing integral equation (62) gives

$$
\begin{aligned}
& \frac{-2}{n} \sum_{\substack{\tilde{j}=1 \\
\tilde{k} \neq \tilde{j}}}^{(M+1) n} \operatorname{Im}\left[\left(\frac{z^{\prime}\left(t_{\tilde{j}}\right)}{\left(z\left(t_{\tilde{k}}\right)-z\left(t_{\tilde{j}}\right)\right)}\right) S^{\prime}\left(t_{\tilde{k}}\right)\right] \\
& +\frac{2}{n} \sum_{\substack{\tilde{j}=1 \\
\tilde{k} \neq \tilde{j}}}^{(M+1) n} \operatorname{Im}\left[\left(\frac{\hat{A}\left(t_{\tilde{j}}\right) S^{\prime}\left(t_{\tilde{j}}\right)}{\left(z\left(t_{\tilde{k}}\right)-z\left(t_{\tilde{j}}\right)\right)}\right)\left(\frac{z^{\prime}\left(t_{\tilde{k}}\right)}{\hat{A}\left(t_{\tilde{k}}\right)}\right)\right] \\
& +\frac{2}{n}\left(\frac{1}{2} \operatorname{Im} \frac{z^{\prime \prime}\left(t_{\tilde{k}}\right)}{z^{\prime}\left(t_{\tilde{k}}\right)}-\operatorname{Im} \frac{\hat{A}^{\prime}\left(t_{\tilde{k}}\right)}{\hat{A}\left(t_{\tilde{k}}\right)}\right) S^{\prime}\left(t_{\tilde{k}}\right)+\frac{1}{n} \sum_{\tilde{j}=1}^{(M+1) n} \chi\left(t_{\tilde{j}}\right) \chi\left(t_{\tilde{k}}\right) S^{\prime}\left(t_{\tilde{j}}\right)=\tilde{\psi}\left(t_{\tilde{k}}\right) .
\end{aligned}
$$

- Equation (64) should change to

$$
(G)_{\tilde{k} \tilde{j}}= \begin{cases}\frac{1}{z\left(t_{\tilde{k}}\right)-z\left(t_{\tilde{j}}\right)}, & k \neq j, \\ 0, & k=j .\end{cases}
$$

- Equation (65) should change to

$$
\begin{aligned}
& \frac{-2}{n} \operatorname{Im}\left[\left(S^{\prime}(\mathbf{t})\left[G z^{\prime}(\mathbf{t})\right]\right)\right]+\frac{2}{n} \operatorname{Im}\left[\left(\frac{z^{\prime}(\mathbf{t})}{\hat{A}(\mathbf{t})}\right)\left[G(\hat{A}(\mathbf{t})) S^{\prime}(\mathbf{t})\right]\right] \\
& +\frac{2}{n}\left(\frac{1}{2} \operatorname{Im} \frac{z^{\prime \prime}(\mathbf{t})}{z^{\prime}(\mathbf{t})}-\operatorname{Im} \frac{\hat{A}^{\prime}(\mathbf{t})}{\hat{A}(\mathbf{t})}\right) S^{\prime}(\mathbf{t})+\mathbf{y}=\tilde{\psi}(\mathbf{t}) .
\end{aligned}
$$

- Under Section 7, Line 24, should change to "Let the $(M+1) n \times 1$ vector $\mathbf{p}$ be the vector $(\hat{A}(\mathbf{t})) S^{\prime}(\mathbf{t})$ or $z^{\prime}(\mathbf{t})$. Let also a be $2 \times(M+1) n$ real vector"

- For the acknowledgment, we wish to add a sentence on the second last line "The authors gratefully acknowledge Prof. Mohamed. M. S. Nasser for helpful discussion related to Section 7." 